\title{
Editorial
}

\section{The Elusive Nature of Truth in Scientific Studies and the Importance of Peer Review}

Constructive, critical peer review is an essential part of the information creation process that has evolved in our society. Journals constitute an essential component of the information dissemination infrastructure because, unlike meetings, the information contained therein is or needs to be subject to the rigors of peer review. Unfortunately, the peer-review process does not always operate as well as it should. For example, Bohannon ${ }^{1}$ submitted an intentionally flawed manuscript, concerning cancer, to 304 journals as part of an experiment to test the peer-review process. This paper was accepted by 157 of those journals. Godlee et al. ${ }^{2}$ submitted a paper with eight deliberate errors (in study design, analysis, and interpretation) to reviewers of the British Medical Journal. Among the 221 respondents, the average number of mistakes detected was two, and some reviewers found no mistakes in the manuscript. We are not perfect, and detection of errors in the peerreview process is not surprising. It does highlight, however, the need both for vigilance (among editors and readers) and an ongoing process for improvement.

Because of the importance of peer review to the quality of Translational Vision Science \& Technology (TVST), we are indebted to the TVST reviewers for the immensely important contributions they have made to the quality of the journal and for the service they have provided to the entire vision research community. On behalf of our Associate Editors, Editors, and readers, I thank each of you sincerely.
Among the highly accomplished individuals who have generously donated their time and expertise for our benefit, a subset have made outstanding contributions due to the exceptional care with which they have reviewed manuscripts. The Editors of TVST have been asked to recognize these individuals by identifying them as "exceptional reviewers." I am pleased to offer special recognition to these individuals as part of our thanks to each of the reviewers for TVST.

TVST is our journal, and it will be as useful a source of information as we make it through the submission of our work and, no less, through the peer review to which that work is subjected.

\section{Marco A. Zarbin}

Editor-in-Chief, TVST

University of Medicine and Dentistry of New Jersey Institute of Ophthalmology and Visual Science Newark, New Jersey

\section{References}

1. Bohannon J. Who's afraid of peer review? Science. 2013;342:60-65.

2. Godlee F, Gale CR, Martyn CN. Effect on the quality of peer review of blinding reviewers and asking them to sign their reports: a randomized controlled trial. JAMA. 1998;280:237-240. 